\title{
Radio-optical properties of extragalactic populations in the VIPERS Survey
}

\author{
A. Zanichelli ${ }^{1}$, L. Gregorini ${ }^{1,2}$, M. Bondi ${ }^{1}$ and the VIPERS Team* \\ ${ }^{1}$ INAF Istituto di Radioastronomia, via Gobetti 101, I-40129 Bologna, Italy, \\ ${ }^{2}$ Dipartimento di Fisica e Astronomia, Università di Bologna, \\ via Ranzani 1, I-40126 Bologna, Italy
}

\begin{abstract}
The radio and optical characteristics of faint radio sources are investigated thanks to the VIPERS spectroscopic and photometric data, and radio data from the VLA FIRST survey at $1.4 \mathrm{GHz}$.
\end{abstract}

The VIMOS Public Extragalactic Redshift Survey (VIPERS, Guzzo et al., 2014) is an ESO Large Program to measure the redshifts of 100.000 galaxies over $\sim 24 \mathrm{deg}^{2}$ of sky in the CFHTLS regions. Based on color pre-selection to remove most of the stars and of galaxies at $\mathrm{z}<0.5$, VIPERS is spectroscopically limited to magnitude $\mathrm{I}_{\mathrm{AB}}<22.5$ and covers the redshift range $0.5<\mathrm{z}<1.2$. A large dataset of ancillary data in various photometric bands is available. The first public data release of $\sim 57.000$ VIPERS redshifts has been done in October 2013 (see http://vipers.inaf.it).

Thanks to VIPERS spectral type classification, the average properties of galaxy types at different cosmic epochs and radio fluxes can be investigated through optical identifications with radio sources in the VLA FIRST catalog (White et al., 1997). For non radio-detected VIPERS galaxies, the stacking of FIRST radio maps allows to push the analysis at very faint radio flux levels.

From the FIRST catalog we classified 1880 radio sources with $\mathrm{S}_{1.4 \mathrm{GHz}} \geqslant 0.77 \mathrm{mJy}$ over the VIPERS area. Among these, 1117 have an optical counterpart in the VIPERS photometric dataset down to $\mathrm{I}_{A B}=24.0, \sim 80 \%$ having either a spectroscopic or a good-quality photometric redshift. The investigation of their radio-optical properties is ongoing.

In parallel, we are conducting the radio stacking analysis for VIPERS galaxies that show no radio emission in the FIRST survey . A preliminary dataset of $\sim 46000$ VIPERS galaxies with good-quality spectroscopic redshift and type classification has been used. First tests show that late spirals/irregulars are detected at increasing redshift, as expected if radio emission in these galaxies is dominated by star formation, while red ellipticals/early spirals are detected at any z, suggesting a probable different origin for radio emission.

The final sample will include the full VIPERS spectroscopic set, allowing us to investigate the different properties of the optical classes at very faint flux limits compared with that of radio-emitting galaxies, and to follow their evolution with redshift.

\section{References}

Guzzo, L., et al. 2014, A\&A 566, 108

White, R.,L., et al. 1997, ApJ 475, 479

\footnotetext{
* The VIPERS Team: U. Abbas, C. Adami, S. Arnouts, J. Bel, J. Blaizot, M. Bolzonella, D. Bottini, E. Branchini, A. Burden, A. Cappi, J. Coupon, O. Cucciati, I. Davidzon, G. De Lucia, S. de la Torre, C. Di Porto, L. Eardley, M. Elmo, P. Franzetti, A Fritz, M. Fumana, B. Garilli, B. Granett, L. Guennou, L. Guzzo (PI), O. Ilbert, A. Iovino, E. Jullo, J. Krywult, V. Le Brun O. Le Fevre, D. Maccagni, A. Marchetti, F. Marulli, K. Malek, C. Marinoni, H. J. McCracken, Y. Mellier, L. Moscardini, T. Moutard, R. Nichol, J. Peacock, W. Percival, M. Polletta, A. Pollo, C. Schimd, M. Scodeggio, V. Scottez, O. Solarz, A. Szalay, L. Tasca, R. Tojeiro, D. Vergani, M. Wilson, A. Zanichelli, G. Zamorani.
} 